\title{
Four versus six cycles of CHOP chemotherapy in combination with six applications of rituximab in patients with aggressive B-cell lymphoma with favourable prognosis (FLYER): a randomised, phase 3, non-inferiority trial
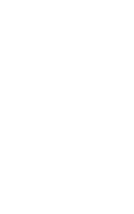

\begin{abstract}
Viola Poeschel*, Gerhard Held*, Marita Ziepert*, Mathias Witzens-Harig, Harald Holte, Lorenz Thurner, Peter Borchmann, Andreas Viardot, Martin Soekler, Ulrich Keller, Christian Schmidt, Lorenz Truemper, Rolf Mahlberg, Reinhard Marks, Heinz-Gert Hoeffkes, Bernd Metzner, Judith Dierlamm, Norbert Frickhofen, Mathias Haenel, Andreas Neubaver, Michael Kneba, Francesco Merli, Alessandra Tucci, Peter de Nully Brown, Massimo Federico, Eva Lengfelder, Alice di Rocco, RalfTrappe, Andreas Rosenwald, Christian Berdel, Martin Maisenhoelder, Ofer Shpilberg, JosifAmam, Konstantinos Christofyllakis, Frank Hartmann, Niels Murawski, Stephan Stilgenbaver, Maike Nickelsen, Gerald Wulf, Bertram Glass, Norbert Schmitz, Bettina Altmann, Markus Loeffler†, Michael Pfreundschuht, for the FLYER Trial Investigatorsł, on behalf of the German Lymphoma Alliance
\end{abstract}

\section{Summary}

Background Six cycles of R-CHOP (rituximab plus cyclophosphamide, doxorubicin, vincristine, and prednisone) are the standard treatment for aggressive B-cell non-Hodgkin lymphoma. In the FLYER trial, we assessed whether four cycles of CHOP plus six applications of rituximab are non-inferior to six cycles of R-CHOP in a population of patients with B-cell non-Hodgkin lymphoma with favourable prognosis.

Methods This two-arm, open-label, international, multicentre, prospective, randomised phase 3 non-inferiority trial was done at 138 clinical sites in Denmark, Israel, Italy, Norway, and Germany. We enrolled patients aged 18-60 years, with stage I-II disease, normal serum lactate dehydrogenase concentration, ECOG performance status $0-1$, and without bulky disease (maximal tumour diameter $<7.5 \mathrm{~cm}$ ). Randomisation was computer-based and done centrally in a 1:1 ratio using the Pocock minimisation algorithm after stratification for centres, stage (I vs II), and extralymphatic sites (no vs yes). Patients were assigned to receive either six cycles of R-CHOP or four cycles of R-CHOP plus two doses of rituximab. CHOP comprised cyclophosphamide $\left(750 \mathrm{mg} / \mathrm{m}^{2}\right)$, doxorubicin $\left(50 \mathrm{mg} / \mathrm{m}^{2}\right)$, and vincristine $\left(1.4 \mathrm{mg} / \mathrm{m}^{2}\right.$, with a maximum total dose of $2 \mathrm{mg}$ ), all administered intravenously on day 1, plus oral prednisone or prednisolone at the discretion of the investigator $(100 \mathrm{mg})$ administered on days 1-5. Rituximab was given at a dose of $375 \mathrm{mg} / \mathrm{m}^{2} \mathrm{of}$ body surface area. Cycles were repeated every 21 days. No radiotherapy was planned except for testicular lymphoma treatment. The primary endpoint was progression-free survival after 3 years. The primary analysis was done in the intention-to-treat population. Safety was assessed in all patients who received at least one dose of assigned treatment. A non-inferiority margin of $-5 \cdot 5 \%$ was chosen. The trial, which is completed, was prospectively registered at ClinicalTrials.gov, NCT00278421.

Findings Between Dec 2, 2005, and Oct 7, 2016, 592 patients were enrolled, of whom 295 patients were randomly assigned to receive six cycles of R-CHOP and 297 were assigned to receive four cycles of R-CHOP plus two doses of rituximab. Four patients in the four-cycles group withdrew informed consent before the start of treatment, so 588 patients were included in the intention-to-treat analysis. After a median follow-up of 66 months (IQR 42-100), 3-year progression-free survival of patients who had four cycles of R-CHOP plus two doses of rituximab was 96\% (95\% CI 94-99), which was 3\% better (lower limit of the one-sided $95 \%$ CI for the difference was $0 \%$ ) than six cycles of R-CHOP, demonstrating the noninferiority of the four-cycles regimen. 294 haematological and 1036 non-haematological adverse events were documented in the four-cycles group compared with 426 haematological and 1280 non-haematological adverse events in the six-cycles group. Two patients, both in the six-cycles group, died during study therapy.

Interpretation In young patients with aggressive B-cell non-Hodgkin lymphoma and favourable prognosis, four cycles of R-CHOP is non-inferior to six cycles of R-CHOP, with relevant reduction of toxic effects. Thus, chemotherapy can be reduced without compromising outcomes in this population.

Funding Deutsche Krebshilfe.

Copyright (C) 2019 Elsevier Ltd. All rights reserved.

\section{Introduction}

In aggressive B-cell non-Hodgkin lymphoma, CHOP (cyclophosphamide, doxorubicin, vincristine, and prednisone) chemotherapy in combination with rituximab is standard of care. ${ }^{1-3}$ Although heterogeneous in its biology, the outcome of the disease can be predicted

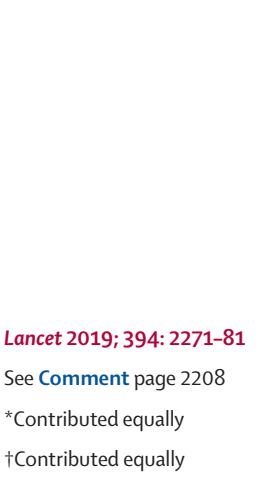

$¥$ A complete list of investigators in the FLYER trial is provided in the appendix pp 3-10

Department of Internal Medicine 1 (Oncology, Hematology,

Clinical Immunology, and Rheumatology), Saarland University Medical School, Homburg/Saar, Germany (V Poeschel MD, LThurner MD, J Amam, K Christofyllakis, F Hartmann MD, N Murawski MD, Prof S Stilgenbauer MD, Prof M Pfreundschuh MDS); Department of Interna Medicine 1, WestpfalzKlinikum, Kaiserslautern, Germany (G Held MD); Institute for Medical Informatics, Statistics and Epidemiology, University Leipzig, Leipzig, Germany (M Ziepert PhD, B Altmann PhD, Prof M Loeffler MD); Department of Interna Medicine V, University of Heidelberg, Heidelberg, Germany (M Witzens-Harig MD); Department of Oncology, Oslo University Hospital, Oslo, Norway (H Holte MD); Department of Hematology and Oncology, University Hospital of Cologne, Cologne, Germany (P Borchmann MD); Department of Internal Medicine III, University Hospital Ulm, Ulm, Germany (A Viardot MD); Department of Internal Medicine II, University Hospital Tuebingen, Tuebingen, Germany (M Soekler MD); Department of 
Internal Medicine III, Klinikum Rechts der Isar der TU München, Munich, Germany (Prof U Keller MD); Department of Medicine III, University Hospital, Munich, Germany

(C Schmidt MD); Department of Hematology and Oncology, Georg August University of Goettingen, Goettingen, Germany (Prof LTruemper MD, Prof G Wulf MD); Department of Internal Medicine I, Klinikum Mutterhaus der Borromaerinnen, Trier, Germany (R Mahlberg MD); Department of Hematology and Oncology, University Medical Center, Freiburg, Germany (R Marks MD); Klinikum Fulda Tumorklinik, Fulda, Germany

(H-G Hoeffkes MD); Department of Hematology and Oncology, Klinikum Oldenburg, Oldenburg, Germany

(B Metzner MD); Department of Internal Medicine II, University Hospital Eppendorf, Hamburg,

Germany (J Dierlamm MD); Department of Internal

Medicine III, Dr Horst-SchmidtKliniken Wiesbaden, Wiesbaden, Germany (N Frickhofen MD); Department of Internal Medicine III, Küchwald Hospital Chemnitz, Chemnitz, Germany

(M Haenel MD); Department of Hematology, Oncology and Immunology, University

Hospital Marburg, Marburg, Germany (Prof A Neubauer MD); Department of Internal Medicine II, City Hospital Kiel, Kiel, Germany (Prof M Kneba MD).

Hematology Azienda Unità Sanitarie Locali-Istituto di Ricovero e Cura a Carattere Scientifico di Reggio Emilia, Reggio Emilia, Italy

(F Merli MD); Hematology Azienda Socio Sanitaria Territoriale Spedali Civili Brescia, Brescia, Italy

(A Tucci MD); Department of Hematology, Rigshospitalet, Copenhagen, Denmark (P de Nully Brown PhD); Dipartimento Chirurgico,

Medico, Odontoiatrico e di

Scienze Morfologiche con Interesse Trapiantologico, Oncologico e di Medicina

Rigenerativa, University of Modena and Reggio Emilia, Modena, Italy

(Prof M Federico MD); Department of Internal Medicine III, University Hospital

\section{Research in context}

\section{Evidence before this study}

Rituximab in combination with six to eight cycles of $\mathrm{CHOP}$ chemotherapy (R-CHOP) has been established as standard treatment for diffuse large B-cell lymphoma. We searched PubMed with the search terms "lymphoma", "DLBCL", "trial", and "rituximab", in English, published between Jan 1, 2002, and Dec 4, 2019. Three randomised trials demonstrated that rituximab improved event-free, progression-free, and overall survival compared with CHOP chemotherapy alone, resulting in a halving of lymphoma-related deaths in these trials. Since then, many trials have failed to improve therapy, suggesting that a plateau of efficacy has been reached with R-CHOP, especially in the subgroup of patients with good prognosis. Prognosis of aggressive non-Hodgkin lymphoma can be established by the International Prognostic Index (IPI), using the clinical parameters of age, tumour stage, serum lactate dehydrogenase concentration, performance status, and number of involved extralymphatic sites. The IPI was established in the pre-rituximab era. Pooled analyses of prospective, randomised trials confirmed the validity of IPI for R-CHOP regimens as well. Patients younger than 60 years without risk factors such as stage III-IV disease, increased serum lactate dehydrogenase

by the clinical parameters of age, tumour stage, serum lactate dehydrogenase concentration, performance status, and number of involved extralymphatic sites. These characteristics have been subsumed in the international prognostic index (IPI), ${ }^{4}$ which reliably separates patients into distinct prognostic subgroups. ${ }^{5}$ For younger patients ( $\leq 60$ years), the age-adjusted model of the IPI (including LDH, stage, and performance status) has been established. Patients without age-adjusted IPI risk factors and without bulky disease (ie, maximum lymphoma diameter $<7.5 \mathrm{~cm}$ ) have a very favourable prognosis with a 3-year progression-free survival of $95 \%$, an event-free survival of $89 \%$, and an overall survival of $98 \%$, when treated with six cycles of combined immunochemotherapy with R-CHOP-like regimens. ${ }^{2,6}$ However, very high cure rates with cytotoxic therapies suggest overtreatment of most patients at least. Accordingly, the benefit in efficacy might come at the cost of unnecessary and potentially severe toxic effects for all patients. Therefore, we hypothesised and tested whether only four cycles of CHOP plus six applications of rituximab are non-inferior to the standard treatment of six cycles of R-CHOP in this population.

\section{Methods \\ Study design and participants}

The investigator-initiated FLYER study was a twoarm, open-label, international, multicentre, prospective, randomised phase 3 trial from 138 clinical sites in Denmark, Israel, Italy, Norway, and Germany. It was coordinated by the German High-grade Non-Hodgkin's concentration, poor performance status, and bulky disease (defined as maximum lymphoma diameter $\geq 7.5 \mathrm{~cm}$ ) had a 3-year progression-free survival of 95\% (95\% Cl 90-99) and an overall survival of $98 \%$ (95-100), when treated with six cycles of $\mathrm{R}$-CHOP or R-CHOP-like regimens in the MInT trial.

\section{Added value of this study}

To our knowledge, this study is the first phase 3 study in aggressive B-cell lymphoma since rituximab was introduced, which showed that the treatment paradigm of six cycles of $\mathrm{R}-\mathrm{CHOP}$ can be changed. It demonstrates that $\mathrm{CHOP}$ chemotherapy can be safely reduced to four cycles in young patients ( $\leq 60$ years) with no risk factor according to the age-adjusted IPI and no bulky disease. Given this excellent outcome, it appears that some patients might be overtreated with six to eight cycles of R-CHOP.

Implications of all the available evidence

We consider these results to be potentially practice changing. Based on the current data, four cycles of $\mathrm{CHOP}$ combined with six doses of rituximab are non-inferior to six cycles of $\mathrm{CHOP}$ combined with six doses of rituximab in young low-risk patients with aggressive B-cell lymphoma.

Lymphoma Study Group, which is now part of the German Lymphoma Alliance. The study was conducted in accordance with the Helsinki declaration. The protocol and its amendments were approved by the ethics committee of each participating centre. Additional information about trial oversight and amendments is provided in the appendix ( $\mathrm{p} 11$ ).

Patients aged 18-60 years were eligible for the study if they had previously untreated biopsy-confirmed aggressive, CD20-positive B-cell lymphoma according to WHO classification of tumours of haemopoietic and lymphoid tissues (third edition, 2001 and fourth edition, 2008) and if they had no risk factor according to ageadjusted IPI (serum lactate dehydrogenase less than upper limit of normal, Eastern Cooperative Oncology Group performance status 0 or 1 , or Ann Arbor stage I or II), and no bulky disease (diameter of single or conglomerate tumour $<7.5 \mathrm{~cm}$ ). Exclusion criteria were CNS involvement or primary CNS lymphoma, marked impairment of cardiac, pulmonary, hepatic, or renal function; white blood cell count less than $2 \cdot 5 \times 10^{3}$ cells per $\mu \mathrm{L}$; initial platelet count less than $100 \times 10^{3}$ cells per $\mu \mathrm{L}$; known hypersensitivity to the medication to be used; known HIV positivity; active hepatitis infection; previous chemotherapy or radiotherapy for past disorder; pregnancy and lactation period; simultaneous participation in other treatment studies; previous immunosuppressive treatment with cytostatics; other concomitant tumour disease or within the past 5 years; MALT lymphoma; planned radiotherapy of extranodal involvement; or inability to comply with study requirements. All patients gave written informed consent. 


\section{Randomisation and masking}

Randomisation was done in a 1:1 ratio using the Pocock minimisation algorithm after stratification for centres, stage (Ann Arbor stage I vs II) and extralymphatic sites (no vs yes). ${ }^{7}$ To ensure balanced group assignment at any time, patients were randomly assigned centrally by a data manager at the study centre (Homburg, Germany) by use of a computer program with an algorithm using a biased coin approach that accounted for previous randomisations. All eligible patients were randomly assigned to receive either six or four 21-day cycles of CHOP chemotherapy given concurrently with six applications of rituximab.

\section{Procedures}

CHOP comprised cyclophosphamide $\left(750 \mathrm{mg} / \mathrm{m}^{2}\right)$, doxorubicin $\left(50 \mathrm{mg} / \mathrm{m}^{2}\right)$, and vincristine $\left(1.4 \mathrm{mg} / \mathrm{m}^{2}\right.$, with a maximum total dose of $2 \mathrm{mg}$ ), all administered intravenously on day 1 , plus oral prednisone or prednisolone at the discretion of the investigator $(100 \mathrm{mg})$ administered on days 1-5. Rituximab was given at a dose of $375 \mathrm{mg} / \mathrm{m}^{2}$ of body surface area. The first cycle of rituximab and first cycle of CHOP started concurrently (denoted R-CHOP). Thus, patients assigned to four cycles of CHOP received the fifth and sixth cycle of rituximab as monotherapy. Cycles were repeated every 21 days.

Lumbar puncture to exclude involvement of the CNS was mandatory in patients with testicular involvement. Prophylaxis for relapse in the CNS was planned to administer to patients with testicular, craniofacial, and upper cervical involvement and patients with Burkitt or Burkitt-like lymphoma. Prophylaxis consisted of four doses of intrathecal methotrexate, $15 \mathrm{mg}$ per dose, administered at day 1 and day 5 of the first and second cycle of R-CHOP, or on day 1 only of the first four cycles of R-CHOP. CNS prophylaxis was stopped by the second amendment of the study protocol on Dec 12, 2011, when an analysis of a large clinical trial showed that intrathecal methotrexate did not lower the incidence of CNS relapse. ${ }^{8}$ Prophylactic radiotherapy with $30 \cdot 6$ Gy to the contralateral testis in case of testicular involvement was mandatory since the second amendment of the study protocol. No radiotherapy was administered to other sites.

Response was assessed after three and six cycles according the 1999 consensus criteria. ${ }^{9}$ First follow-up examination was done 3 months after the restaging after six cycles. Follow-up examinations thereafter took place during the initial 2 years every 3 months, in years 3-5 every 6 months and then subsequently on an annual basis. Follow-up examinations consisted of a clinical examination, laboratory analysis, imaging techniques, and documentation of remission status and of therapyinduced disorders, including secondary neoplasia.

\section{Outcomes}

Progression-free survival at 3 years was the primary endpoint, which was defined as the time from randomisation until one of the following events had occurred: progression during therapy, progressive disease after partial response, no change, unknown status at the end of study therapy, relapse after complete response or unconfirmed complete response or death from any cause, whichever came first. Response was assessed by local investigators. The key secondary endpoints were eventfree survival and overall survival. Event-free survival was defined as the time from randomisation until one of the following events had occurred: progression during therapy, partial response, no change, unknown status at the end of study therapy, relapse after complete response or unconfirmed complete response, death from any cause; or additional treatment, whichever came first. Overall survival was defined as the time from randomisation until death from any cause. If no events occurred, patients were censored at the time of the last available information.

Other secondary endpoints were rate of complete remissions and progressive disease, safety (adverse events, serious adverse events, rate of secondary neoplasia, selected laboratory parameters, including leucocytes, thrombocytes, and haemoglobin), adherence to protocol (duration of cycles, cumulative dose, and dose intensity) and health-economic aspects (using the cumulative dose of chemotherapy drugs and rituximab). Other endpoints of health economic aspects (days in hospital, total number of days on which antibiotics were administered, total numbers of erythrocyte and platelet concentrates, and measures provided to treat serious adverse events) will be reported elsewhere. Adverse events were classified in accordance with the German version of the NCI common toxicity criteria prepared by the Deutsche Krebsgesellschaft (German Cancer Society). Severe adverse events had to be reported within 1 working day (if the event occurred during therapy) or within 10 working days (if the event occurred during the follow-up phase).

\section{Statistical analysis}

The trial was planned to show the non-inferiority of four cycles of R-CHOP plus two cycles of rituximab monotherapy versus six cycles of R-CHOP.

Progression-free survival at 3 years was selected as primary endpoint, assuming a rate of $93 \%$ for six cycles of R-CHOP and no difference between groups. With a prespecified non-inferiority margin of $-5 \cdot 5 \%$ and a $10 \%$ dropout rate, 592 patients are required to reach a power of $80 \%$, at a significance level of $5 \%$ (one-sided). ${ }^{10}$ The primary and secondary survival endpoints were analysed with the Kaplan-Meier method. To demonstrate non-inferiority we used the difference between the 3 years progression-free survival rate of four cycles versus six cycles of CHOP, each combined with six cycles of rituximab, calculated the one-sided 95\% CI using the Kaplan-Meier estimates and Greenwood's estimates of the corresponding variance, and established whether it lies entirely on the positive side of the prespecified noninferiority margin of $-5 \cdot 5 \% .^{11}$ The primary analysis was
Mannheim, Mannheim Germany (E Lengfelder MD); Department of Translational and Precision Medicine Sapienza University of Rome, Rome, Italy (A di Rocco PhD); Department of Internal Medicine II, Evangelisches Diakonie-Krankenhaus, Bremen, Germany (RTrappe MD); Institute of Pathology, University of Wuerzburg, and Comprehensive Cancer Center Mainfranken, Wuerzburg, Germany (Prof A Rosenwald MD); Department of Radiooncology Saarland University Medical School, Homburg/Saar, Germany (C Berdel MD); Department of Oncology, University Hospital of North Norway, Tromsø, Norway (Martin Maisenhoelder MD); Department of Hematology, Rabin Medical Center, Beilinson Hospital, Petah-Tiqwa, Israe (Prof O Shpilberg MD); and Department of Hematology Oncology and Stem Cell Transplantation, Asklepios Klinik St Georg, Hamburg, Germany (M Nickelsen MD B Glass MD, N Schmitz MD)

SProf M Pfreundschuh died in March, 2018

Correspondence to: Dr Viola Poeschel, Department of Internal Medicine I, Saarland University Medical School, 66421 Homburg/Saar, Germany viola.poeschel@uks.eu or

Prof Gerhard Held, Department of Internal Medicine 1 , Westpfalz-Klinikum, 67655 Kaiserslautern, Germany gheld@westpfalz-klinikum.de See Online for appendix 


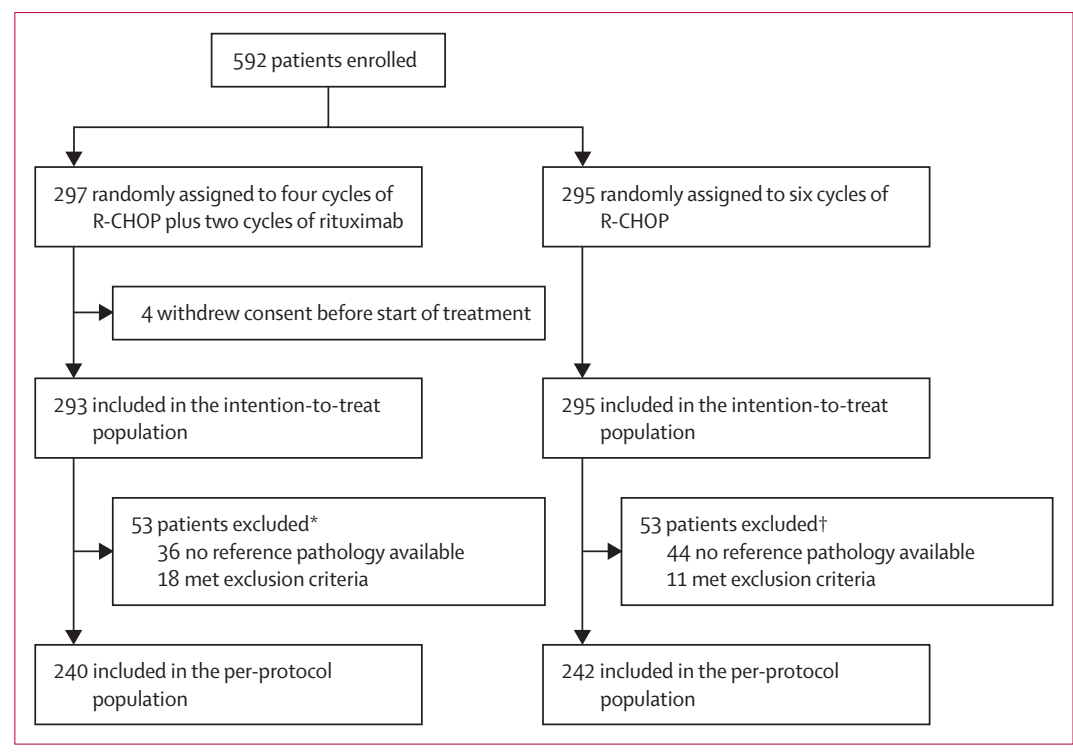

Figure 1: Trial profile

$\mathrm{R}-\mathrm{CHOP}=$ rituximab plus cyclophosphamide, doxorubicin, vincristine, and prednisone. IPI=international prognostic index. *One patient was excluded for both reasons stated. †Two patients were excluded for both reasons stated.

\begin{tabular}{|c|c|c|}
\hline & $\begin{array}{l}\text { Four cycles of } \\
\text { R-CHOP plus } \\
\text { two cycles of } \\
\text { rituximab group } \\
(n=293)\end{array}$ & $\begin{array}{l}\text { Six cycles of } \\
\text { R-CHOP group } \\
(n=295)\end{array}$ \\
\hline \multicolumn{3}{|l|}{ Sex } \\
\hline Female & $118(40 \%)$ & $116(39 \%)$ \\
\hline Male & $175(60 \%)$ & $179(61 \%)$ \\
\hline \multicolumn{3}{|l|}{ Age } \\
\hline Median (IQR) & $49(40-55)$ & $47(41-54)$ \\
\hline Range & $18-60$ & $19-60$ \\
\hline $\begin{array}{l}\text { Serum lactate dehydrogenase } \\
\text { greater than upper limit of normal }\end{array}$ & 0 & 0 \\
\hline $\begin{array}{l}\text { Eastern Cooperative Oncology Group } \\
\text { performance status }>1\end{array}$ & 0 & 0 \\
\hline Stage III or IV* & $2(1 \%)$ & $4(1 \%)$ \\
\hline \multicolumn{3}{|c|}{ Age-adjusted International Prognostic Index } \\
\hline 0 & $291(99 \%)$ & $291(99 \%)$ \\
\hline $1^{*}$ & $2(1 \%)$ & $4(1 \%)$ \\
\hline \multicolumn{3}{|l|}{ Stage } \\
\hline I & $174(59 \%)$ & $172(58 \%)$ \\
\hline ॥ & $117(40 \%)$ & $119(40 \%)$ \\
\hline $1 I^{*}$ & $1(<1 \%)$ & $2(1 \%)$ \\
\hline $\mathrm{IV}^{*}$ & $1(<1 \%)$ & $2(1 \%)$ \\
\hline Bulky disease* & $1(<1 \%)$ & $1(<1 \%)$ \\
\hline Extralymphatic involvement & $95(32 \%)$ & $96(33 \%)$ \\
\hline B-symptoms & $27(9 \%)$ & $9(3 \%)$ \\
\hline Diffuse large B-cell lymphoma & $252(86 \%)$ & $247(84 \%)$ \\
\hline Not otherwise specified & $163(56 \%)$ & $172(58 \%)$ \\
\hline Centroblastic & $73(25 \%)$ & $62(21 \%)$ \\
\hline Immunoblastic & $4(1 \%)$ & $6(2 \%)$ \\
\hline Anaplastic large cell & $5(2 \%)$ & $4(1 \%)$ \\
\hline T-cell-rich B-cell lymphoma & $4(1 \%)$ & $3(1 \%)$ \\
\hline $\begin{array}{l}\text { Primary mediastinal B-cell } \\
\text { lymphoma }\end{array}$ & $3(1 \%)$ & 0 \\
\hline Follicular lymphoma IIIB & $17(6 \%)$ & $9(3 \%)$ \\
\hline $\begin{array}{l}\text { Follicular lymphoma IIIB plus diffuse } \\
\text { large B-cell lymphoma }\end{array}$ & $17(6 \%)$ & $23(8 \%)$ \\
\hline Burkitt lymphoma & $2(1 \%)$ & $2(1 \%)$ \\
\hline Burkitt-like lymphoma & $1(<1 \%)$ & $3(1 \%)$ \\
\hline Aggressive marginal zone lymphoma & $1(<1 \%)$ & $1(<1 \%)$ \\
\hline $\begin{array}{l}\text { High-grade B-cell lymphoma not } \\
\text { otherwise specified }\end{array}$ & $3(1 \%)$ & $10(3 \%)$ \\
\hline \multicolumn{3}{|c|}{$\begin{array}{l}\text { Data are } \mathrm{n}(\%) \text { unless otherwise specified. } \mathrm{R}-\mathrm{CHOP}=\text { rituximab with } \\
\text { cyclophosphamide, doxorubicin, vincristine, and prednisone. }{ }^{*} \text { Caused by } \\
\text { retrospective changes, which were done by the investigators after data clearing. }\end{array}$} \\
\hline
\end{tabular}

The corresponding authors had full access to all the data in the study and the final responsibility for the decision to submit for publication.

\section{Results}

From Dec 2, 2005, to Oct 7, 2016, 592 patients were enrolled and randomly assigned to either four cycles of R-CHOP plus two cycles of rituximab $(n=297)$ or six cycles 
of R-CHOP $(n=295)$. Four patients in the four-cycles group withdrew their informed consent before the start of treatment, so 588 patients were included in the intention-to-treat analysis (figure 1).

Baseline characteristics were well balanced except B symptoms, with 27 (9\%) of 293 patients in the four-cycles group and nine (3\%) patients in the six-cycles group $(\mathrm{p}=0 \cdot 002)$ initially presenting with $\mathrm{B}$ symptoms (table 1).

According to the primary pathology report, 499 (85\%) of 588 patients had diffuse large B-cell lymphoma or one of its subtypes (table 1). Randomisation was done if diagnosis of the primary pathology was available. Reference pathology was done in 508 (86\%) of 588 patients (appendix p 24).

The first planned interim analysis was done on July 5, 2013, in 408 patients. During the course of the trial, the efficacy endpoints for patients with aggressive lymphoma were internationally harmonised and published. ${ }^{12}$ Additionally, progression-free survival was established as the preferred primary endpoint for clinical trials in aggressive lymphoma. ${ }^{12}$ In the interim analysis of July, 2013, the initially planned primary endpoint of 3-year event-free survival showed a lower rate than assumed in the protocol, but the 3-year progression-free survival rate met that used for sample size calculation. The DSMB agreed to an amendment on 30 March, 2015, using progression-free survival as the primary endpoint.

Data cutoff for the final analysis was June 18, 2018. Median follow-up time was 66 months (IQR 42-100) for progression-free survival.

The median duration of chemotherapy from day one of the first cycle until day one of the last cycle of chemotherapy was 63 days (IQR 62-64) in the four cycle group and 105 days (105-107) in the six-cycles group. In both treatment groups the median duration of rituximab immunotherapy was 106 days (IQR 106-107 for four cycles and 106-108 for six cycles; appendix pp 16-17).

11 patients with testicular involvement were included (five in the four-cycles group and six in the six-cycles group; appendix p 26). Prophylactic radiotherapy with 30.6 Gy to the contralateral testis in case of testicular involvement was mandatory since the second amendment of the study protocol (appendix p 23). No radiotherapy was planned to be administered to other sites. Additional radiotherapy to the initially involved extralymphatic sites was given to 18 patients (seven in the four-cycles group and 11 in the six-cycles group; appendix p 27).

After a median follow-up of 66 months, 3-year progression-free survival was $96 \%$ (95\% CI 94-99) with four cycles of R-CHOP plus two cycles of rituximab versus 94\% (91-97) with six cycles of R-CHOP (figure 2A). The

Figure 2: Progression-free survival (A), event-free survival (B), and overal survival $(C)$ in the intention-to-treat population

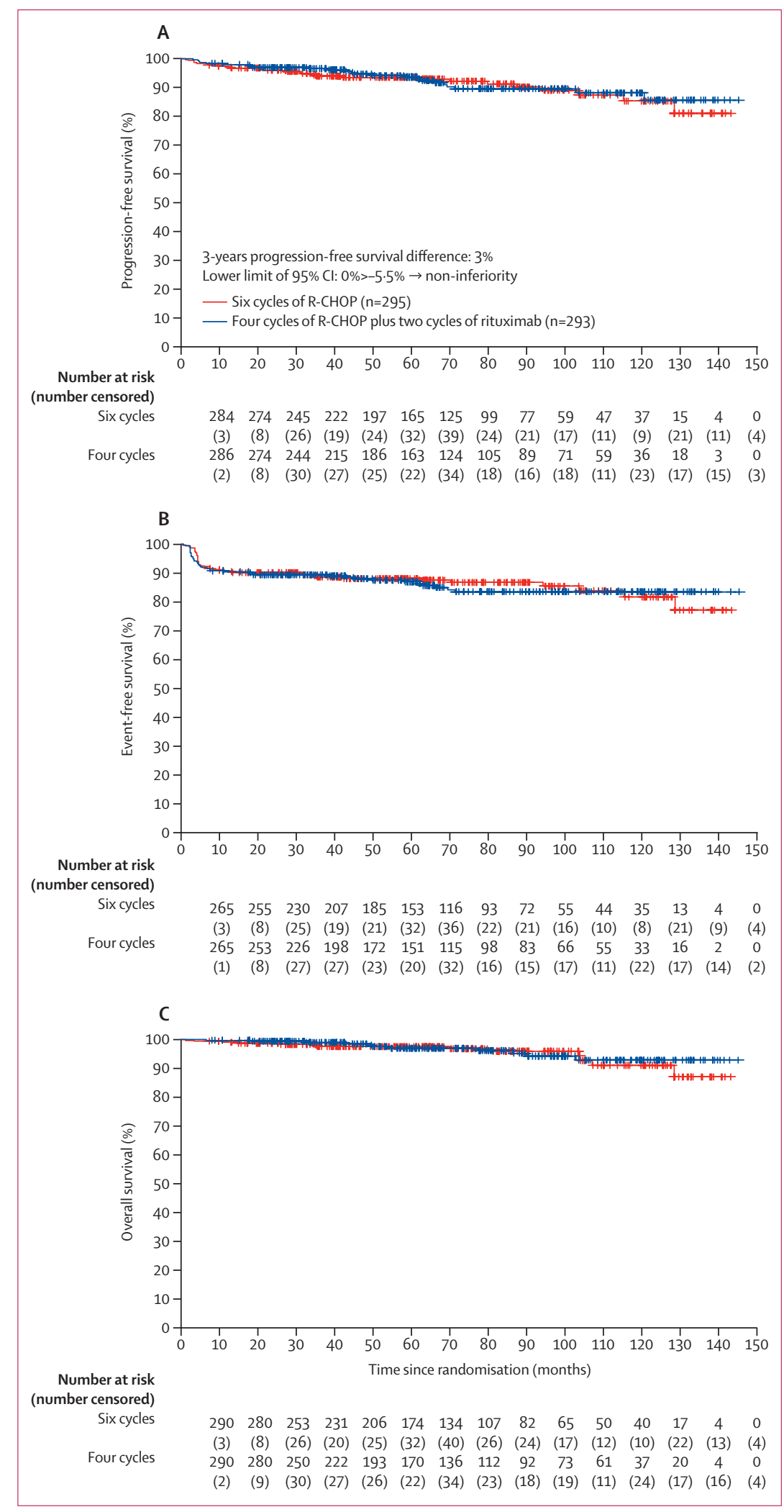




\begin{tabular}{|c|c|c|}
\hline & $\begin{array}{l}\text { Four cycles of } \\
\text { R-CHOP plus } \\
\text { two cycles of } \\
\text { rituximab } \\
\text { group }(n=293)\end{array}$ & $\begin{array}{l}\text { Six cycles of } \\
\text { R-CHOP } \\
\text { group } \\
(n=295)\end{array}$ \\
\hline $\begin{array}{l}\text { Complete response or unconfirmed } \\
\text { complete response }\end{array}$ & $\begin{array}{l}267 \\
(91 \% ; 87-94)\end{array}$ & $\begin{array}{l}271 \\
(92 \% ; 88-95)\end{array}$ \\
\hline Partial response* & $8(3 \%)$ & $11(4 \%)$ \\
\hline No change & 0 & $1(<1 \%)$ \\
\hline Progressive disease & $3(1 \%)$ & $3(1 \%)$ \\
\hline Not evaluated or missing data $†$ & $15(5 \%)$ & $9(3 \%)$ \\
\hline $\begin{array}{l}\text { Relapse after complete response or } \\
\text { unconfirmed complete response }\end{array}$ & $\begin{array}{c}11 / 267 \\
(4 \% ; 2-7)\end{array}$ & $\begin{array}{c}13 / 271 \\
(5 \% ; 3-8)\end{array}$ \\
\hline Relapse after partial response & $2 / 8(25 \%)$ & $2 / 11(18 \%)$ \\
\hline Relapse after no change & 0 & $1 / 1(100 \%)$ \\
\hline $\begin{array}{l}\text { Relapse after not evaluated or missing } \\
\text { data }\end{array}$ & $3 / 15(20 \%)$ & $2 / 9(22 \%)$ \\
\hline \multicolumn{3}{|c|}{$\begin{array}{l}\text { Data are } \mathrm{n}(\% ; 95 \% \mathrm{Cl}), \mathrm{n}(\%), \mathrm{n} / \mathrm{N}(\% ; 95 \% \mathrm{Cl}) \text {, or } \mathrm{n} / \mathrm{N}(\%) \text {. } \mathrm{R}-\mathrm{CHOP}=\text { rituximab } \\
\text { with cyclophosphamide, doxorubicin, vincristine, and prednisone. }{ }^{*} \text { Five patients } \\
\text { with partial response in the four-cycles group and six in the six-cycles group had } \\
\text { complete response or unconfirmed complete response but received additional } \\
\text { treatment outside study protocol. } † \text { Six patients in the six-cycles group } \\
\text { prematurely discontinued chemotherapy, including two therapy-associated } \\
\text { deaths in the six-cycles group, without having a response assessment; } 18 \text { patients } \\
\text { started subsequent treatment without having a response assessment. }\end{array}$} \\
\hline
\end{tabular}

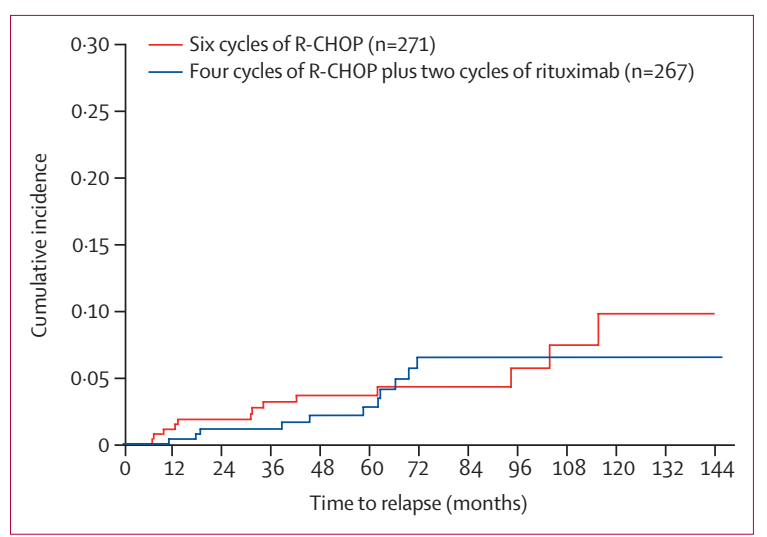

Figure 3: Cumulative incidence of relapse

absolute difference between groups in 3-year progression-free survival was 3\% (lower limit of the onesided $95 \%$ CI $0 \%$ ). Thus, the $95 \%$ CI is on the positive side of the prespecified non-inferiority margin of $-5.5 \%$, demonstrating the non-inferiority of four cycles of R-CHOP plus two cycles of rituximab versus six cycles of R-CHOP.

A detailed description of absolute and relative dose and relative dose intensity is in the appendix (appendix pp 18-19). Protocol deviations occurred in six patients in the six-cycles group and eight in the four-cycles group (nine were rituximab deviations and seven were chemotherapy deviations (appendix p 25).

In both treatment groups, remission rates were high. At the end of therapy, 267 (91\%) of 293 patients in the four-cycles group versus 271 (92\%) of 295 patients in the six-cycles group had a complete response or unconfirmed complete response (table 2). Eight (3\%) patients in the four-cycles group versus $11(4 \%)$ in the six-cycles group had a partial response. None of the patients in the four-cycles group and one $(<1 \%)$ patient in the six-cycles group had no change. In both groups, three (1\%) patients progressed while on therapy. A more detailed description of patients with partial response or an unknown response is provided in the appendix (pp 45-47).

3-year event-free survival was $89 \%$ (95\% CI $86-93)$ in the four-cycles group versus $89 \%$ (85-92) in the six-cycles group (figure 2B). 3-year overall survival was $99 \%(98-100)$ in the four-cycles group versus $98 \%$ (96-99) in the six-cycles group (figure 2C).

In a post-hoc analysis, median time from biopsy to start of prephase treatment was 23 days (IQR 15-31), to first application of rituximab it was 32 days (23-43), and to first cycle of CHOP it was 32 days (23-43; appendix p 15).

In a post-hoc analysis of patients with progression-free survival who had complete and partial remission at interim restaging, progression-free survival did not differ between the treatment groups (appendix p 20). In posthoc analyses of progression-free survival in patients who were recruited in the first half of the trial and those recruited in the last half, results were similar between treatment groups (appendix pp 21-22). The multivariate analysis for progression-free survival comparing four cycles with six cycles after adjusting for strata and B symptoms showed a hazard ratio of $0.9(95 \% \mathrm{Cl}$ $0 \cdot 5-1 \cdot 6 ; \mathrm{p}=0 \cdot 810$; appendix $\mathrm{p} 28$ ).

The long median follow-up allowed us to estimate 5 -year outcomes in post-hoc analyses. Progression-free survival was $94 \%$ (95\% CI 91-97) in the four-cycles group versus $94 \%$ (91-96) in the six-cycles group. 5-year eventfree survival was $87 \%(83-91)$ in the four-cycles group versus $88 \%$ (84-92) in the six-cycles group. 5-year overall survival was $97 \%$ (94-99) in the four-cycles group versus $98 \%(96-100)$ in the six-cycles group.

Multivariate analysis comparing overall survival between the treatment groups after adjusting for strata and B symptoms showed a hazard ratio of $0 \cdot 9(95 \% \mathrm{Cl}$ $0 \cdot 4-1 \cdot 9 ; p=0 \cdot 722$; appendix $p$ 28). Cause of death was related to progression or relapse of lymphoma in five patients in the four-cycles group versus seven patients in the six-cycles group (appendix p 28).

The per-protocol analysis of 482 patients who fulfilled inclusion criteria showed similar results for 3-year progression-free survival, which was $98 \%$ (95\% CI 96-100) in the four-cycles group versus $94 \%$ (91-97) in the six-cycles group.

40 patients progressed or relapsed. The relapse rates for patients with complete response or unconfirmed complete response at the end of therapy were similar in both treatment groups (table 2; appendix pp 29-44). Three of 11 patients with testicular involvement relapsed (appendix p 26). Four (17\%) of 24 relapses after complete 
response or unconfirmed complete response occurred in the first year after enrolment. Eight (33\%) occurred in the 2 years after study inclusion but continued to occur with longer follow-up in both groups (figure 3 ). No patient relapsed in the CNS.

The safety population included 293 patients in the fourcycles group and 295 patients in the six-cycles group. Fewer adverse events were documented during therapy in the four-cycles group, with 294 haematological adverse events, than in the six-cycles group, with 426 haematological adverse events (table 3).

Fewer non-haematological adverse events occurred in the four-cycles group (1036 events) than in the six-cycles group (1280 events; table 3). 48 serious adverse events occurred in the four-cycles group compared with 45 in the six-cycles group.

In the four-cycles group, 116 infections were reported, 22 of which were grade 3 or grade 4.156 infections were reported in the six-cycles group, of which 23 were grade 3 or grade 4. Two patients, both in the six-cycles group, died during study therapy (table 3 ). Seven cardiac events as atrial fibrillation, heart failure, and coronary artery disease have been reported during therapy and followup: four events in the four-cycles group and three events in the six-cycles group.

$18(6 \%)$ of 293 patients in the four-cycles group versus $14(5 \%)$ of 295 in the six-cycles group developed a secondary neoplasm (appendix p 48). Cause of death was related to secondary neoplasm in three $(1 \%)$ patients in the four-cycles group versus four (1\%) patients in the six-cycles group (appendix p 28).

\section{Discussion}

Young patients with aggressive B-cell non-Hodgkin lymphoma and no IPI risk factors, such as the population included in our study, have an excellent prognosis. We showed that reduction of chemotherapy to four cycles of R-CHOP plus two cycles of rituximab from the standard six cycles of R-CHOP results in similar progression-free survival after 3 years, which was the primary endpoint of this study, demonstrating the non-inferiority of four cycles compared with the six cycles. Also, no difference in event-free survival or overall survival was detected, neither after 3 years nor after 5 years of followup. Reliability of the results is provided by the multicentre setting of this large international trial. In these patients, four cycles of CHOP in combination with six applications of rituximab are non-inferior in eradicating the malignant lymphoma clone. This conclusion is confirmed by the observation of similar responses and relapse rates.

To provide benefit to patients with aggressive B-cell non-Hodgkin lymphoma with such a favourable risk profile, our trial studied the reduction of cytostatic drugs rather than rituximab, which has only minor toxic effects. ${ }^{1-3}$ In fact, some evidence suggests increased efficacy when rituximab is administered over a prolonged period of time in aggressive non-Hodgkin lymphoma.

\begin{tabular}{|c|c|c|c|c|}
\hline & \multicolumn{2}{|c|}{$\begin{array}{l}\text { Four cycles of R-CHOP plus } \\
\text { two cycles of rituximab group } \\
(n=293)\end{array}$} & \multicolumn{2}{|c|}{$\begin{array}{l}\text { Six cycles of R-CHOP group } \\
(n=295)\end{array}$} \\
\hline & Any grade & Grades 3-4 & Any grade & Grades 3-4 \\
\hline Leucocytopenia* & 171 & 80 & 237 & 110 \\
\hline Anaemia $†$ & 107 & 2 & 172 & 8 \\
\hline Thrombocytopenia $\ddagger$ & 16 & 5 & 17 & 7 \\
\hline Non-haematological adverse event & 1036 & 52 & 1280 & 71 \\
\hline Infection & 116 & 22 & 156 & 23 \\
\hline Paresthesia & 342 & 16 & 370 & 14 \\
\hline Nausea & 221 & 6 & 319 & 12 \\
\hline Vomiting & 61 & 1 & 117 & 7 \\
\hline Mucositis & 80 & 1 & 105 & 3 \\
\hline Constipation & 100 & 4 & 69 & 2 \\
\hline Mood alteration & 59 & 1 & 60 & 0 \\
\hline Diarrhoea & 33 & 0 & 40 & 6 \\
\hline Arrhythmia & 8 & 1 & 24 & 0 \\
\hline Allergy & 16 & 0 & 19 & 3 \\
\hline Paraplegia &.. &.. & 15 & 1 \\
\hline Therapy-associated deaths &.. &.. & 29 &.. \\
\hline \multicolumn{5}{|c|}{$\begin{array}{l}\text { Leucocytopenia, anaemia, and thrombocytopenia were documented only during the chemotherapy. R-CHOP=rituximab } \\
\text { with cyclophosphamide, doxorubicin, vincristine, and prednisone. *Relevant leucocyte counts between cycles, on } \\
\text { days } 11-14 \text {, were available in } 192 \text { of } 1161 \text { cycles in the four-cycles group and } 267 \text { of } 1746 \text { cycles in the six-cycles group. } \\
\text { tHaemoglobin values were documented in } 839 \text { of } 1161 \text { cycles in the four-cycles group and in } 1207 \text { of } 1746 \text { cycles in the } \\
\text { six-cycles group. } ¥ \text { Thrombocyte counts were documented in } 848 \text { of } 1161 \text { cycles in the four-cycles group and } 1205 \text { of } \\
1746 \text { cycles in the six-cycles group. SAfter the first application of } 15 \mathrm{mg} \text { intrathecal methotrexate administered as CNS } \\
\text { prophylaxis in a patient with stage II disease and testicular involvement. } 9 \text { Two patients, both in the six-cycles group, } \\
\text { died during study therapy; one patient died after the second cycle of R-CHOP from pneumonia caused by influenza } \\
\text { H1N1 virus; the other patient died after the fifth cycle of R-CHOP from an atypical pneumonia after having herpes zoster } \\
\text { ophthalmicus. }\end{array}$} \\
\hline
\end{tabular}

Rituximab maintenance tested in the NHL13 trial ${ }^{14}$ increased event-free survival as well as progression-free survival in the subgroup of male patients. In elderly patients, a phase 2 trial $^{15}$ demonstrated superior survival after six cycles of CHOP-14 in combination with an extended rituximab exposure time compared with a historical control. Thus, we do not recommend less than six applications of rituximab, which led to excellent outcomes in the pivotal MInT trial. ${ }^{2}$

A different strategy in stage I or II aggressive B-cell non-Hodgkin lymphoma based on three cycles of R-CHOP plus involved-field radiotherapy was investigated in a smaller phase 2 trial. ${ }^{16}$ The SWOG 0014 study included patients with stage I or II disease, but with a more unfavourable prognostic profile with at least one adverse risk factor for non-bulky stage II disease, age older than 60 years, WHO performance status of 2, or increased serum lactate dehydrogenase. The treatment resulted in a progression-free survival of $93 \%$ and an overall survival of $95 \%$ at 2 years and a progression-free survival of $88 \%$ and an overall survival of $92 \%$ at 4 years. ${ }^{16}$ However, in light of a 3 -year overall survival of $99 \%$ after four cycles of R-CHOP plus rituximab in our trial, it seems unlikely that radiotherapy is providing an improvement in outcome in patients with such a 
favourable prognostic profile who were included in our phase 3 trial. Indeed, a phase 3 trial in non-bulky stage I or II diffuse large B-cell lymphoma did not show any improvement in event-free survival and overall survival with additive involved-field radiotherapy after R-CHOP compared with R-CHOP alone. ${ }^{17}$ Thus, radiotherapy seems to not be relevant a priori, but might be confined to a minority of patients who are responding poorly. FDG-PET-CT scan has become standard in response assessment of lymphoma. ${ }^{18}$ When FDG-PET scanning is performed, it is more prognostic the later it is applied during the course of immunochemotherapy. ${ }^{19}$ However, the positive predictive value of the method is low, ranging only from $20-74 \%$, which makes it difficult to predict a relapse for an individual patient. ${ }^{20}$ But it remains a valid tool to allocate patients into subgroups with different outcomes. Radiotherapy might be given to those who are still positive in FDG-PET after two to four cycles of R-CHOP. Radiotherapy is feasible, because in these patients with a maximal Ann Arbor stage II disease and the absence of bulky disease, only small irradiation volumes will be needed. Indeed, when patients aged 61-80 years with an age-adjusted IPI of 0 and a positive FDG-PET after four cycles of R-CHOP-14 are treated with six cycles of R-CHOP-14 followed by modified involvedsite radiotherapy, they have similar progression-free survival and overall survival to those patients with a negative FDG-PET scan. ${ }^{21}$ Thus, positive FDG-PET-CT scan potentially might identify patients whose inferior prognosis can be compensated for by radiotherapy. These results have been obtained from the interim analysis of a still ongoing and recruiting clinical trial (NCT01478542). Therefore, the final results have to be awaited before this strategy can be estimated as safe and effective with the appropriate methodical and scientific validity.

We did not include patients older than 60 years, because age is an independent risk factor for inferior survival. ${ }^{4,5}$ The additional risk factors of the IPI still accurately separate elderly patients to distinct prognostic subgroups and interim FDG-PET predicts survival independent of the IPI. ${ }^{22}$ The result of two ongoing clinical trials (LNH 2009-1B [NCT01285765] and OPTIMAL $>60$ [NCT01478542]), which are testing FDGPET-based, response-adapted reduction of chemotherapy in patients with good prognosis according to IPI might answer the question of whether the number of cycles of CHOP chemotherapy can also be reduced in patients older than 60 years.

We also did not include patients with bulky disease, which is associated with inferior prognosis in a population of young patients with an otherwise good prognosis. ${ }^{6}$ Primary mediastinal B-cell lymphoma is a distinct pathogenetic subtype of aggressive lymphoma, which typically presents in young patients with an isolated manifestation as bulky anterior mediastinal mass. ${ }^{23}$ Only three patients with primary mediastinal B-cell lymphoma have been included, because bulky disease, defined as maximal tumour diameter of $7.5 \mathrm{~cm}$ or more was an exclusion criterion. Therefore, our conclusion can't be extended to this particular entity.

Relapse rates after complete response or unconfirmed complete response were low in patients included in this trial. Relapse rate in the low number of included patients with testicular involvement was similar to that reported before. ${ }^{24}$ Notably, cumulative incidence of relapse increased linearly during follow-up, similar to indolent non-Hodgkin lymphoma..$^{25}$ This pattern differs from that observed in an unselected population of patients with diffuse large B-cell lymphoma, where $70 \%$ of relapses occurred within the first year after diagnosis with a continued declining rate as the time from diagnosis increased. ${ }^{26}$ The observation suggests that lymphoma, which present with good prognostic features without risk factors according to the ageadjusted IPI, and whose malignant clone is not eradicated by R-CHOP, might have a different biological background driving this unique pattern of relapse. A thorough and accurate molecular analysis of the lymphoma at primary diagnosis and late relapse is warranted to decipher mechanisms of chemotherapy resistance, evolution of relapse, or eventually, de-novo lymphoma genesis.

Notably, no progression or relapse in the CNS occurred among the 588 patients of the FLYER study. This observation confirms a recently developed risk model for CNS relapse of diffuse large B-cell lymphoma. ${ }^{27}$ The so-called CNS-IPI consists of the IPI risk factors in addition to involvement of kidneys or adrenal glands. The CNS-IPI predicts a risk for CNS relapse of $0 \%$ for the population of the FLYER study, which included only patients with no risk factor according to the IPI and CNS-IPI (only one of 588 patients had involvement of the kidney). Therefore, the prediction of the CNS-IPI is in line with the results observed. Also, patients presenting such a favourable risk profile do not need a CNS-directed prophylaxis.

We observed less acute haematological and nonhaematological toxicities with the reduced number of chemotherapy cycles, as expected, but no relevant differences in secondary neoplasia. This is probably because of the very low incidence of second primary malignancies in both treatment groups. However, to assess long-term toxicity of curative immunochemotherapy, a longer follow-up than that in our study is necessary.

Our study has some limitations. The recruitment extended over a period of more than 10 years. However, treatment did not change over time, but was confirmed because numerous randomised trials did not improve on R-CHOP. ${ }^{28-30}$ Also, we found no evidence for a treatment by time interaction (appendix pp 21-22). The WHO classification of lymphoma was modified three times since the study was started: the WHO classification of tumours of haemopoietic and lymphoid 
tissues revised fourth edition in 2017, made it essential to assign diffuse large B-cell lymphoma-not otherwise specified according to its cell of origin, either germinal centre B-cell-like or activated B-cell-like. However, the concept of cell of origin does not affect R-CHOP as standard of care in aggressive B-cell lymphoma. ${ }^{30,31}$ Response assessment of lymphoma changed, making an FDG-PET scan mandatory to distinguish between complete response and partial response., ${ }^{9.18}$ But the changes would not have affected progression-free survival, which was the primary endpoint of our study, nor overall survival. Therefore, these modifications, which were introduced to the standard care of patients with lymphoma, have no influence on the primary endpoint and conclusions of our study. Biological risk factors need special consideration, especially translocations of $M Y C$, which might occur in combination with $B C L 2$ or BCL6 translocation as so-called double-hit or triple-hit lymphoma. It requires FISH testing and was implemented in the fourth edition of the WHO classification of tumours of haemopoietic and lymphoid tissues, which was published when accrual of the FLYER trial was already finished. When $M Y C$ is rearranged with an immunoglobulin gene it confers to an inferior prognosis. ${ }^{32}$ We do not know whether in patients with an MYC translocation reduction of cycles of chemotherapy is safe. But applying an age-adjusted IPI of 0 as an inclusion criterion and bulky disease as an exclusion criterion results in a 3-year progressionfree survival of $96 \%$ and overall survival of $99 \%$ after four cycles of R-CHOP with six applications of rituximab, clearly selecting patients with good prognosis and excluding those with poor prognosis. A detailed analysis of biological risk factors is warranted, ongoing, and will be reported. Another limitation is that quality of life was not assessed systematically. However, toxicity was reduced, which is associated with better quality of life. Besides reduced toxicity, returning to an unrestricted family and professional life quicker is a very important benefit.

The strengths of the study are its clear phase 3 design. The large number of patients is an adequate sample size to address the objective. Patients have been recruited at 138 sites across five countries, represented by private practice-based oncologists, primary, secondary, tertiary, and university hospitals, reflecting a real world scenario of the health-care systems. Furthermore, the analysis of primary and secondary endpoints provides definite results, also in predefined and post-hoc subgroups.

In conclusion, treatment of young patients with aggressive B-cell lymphoma and a favourable risk profile can be reduced to four cycles of CHOP chemotherapy plus six cycles of rituximab without compromising efficacy.

Contributors

MP was the study chairman and designed and oversaw the study. VP and $\mathrm{GH}$ oversaw the study and contributed to study design, data monitoring, data interpretation, and writing and approval of the report. MZ and ML did the statistical analysis and contributed to study design, data interpretation, and writing and approval of the report. AR coordinated the reference pathology. CB coordinated and assessed the imaging review. OS was the principal investigator in Israel and recruited patients. $\mathrm{HH}$ was the principal investigator in Norway and recruited patients. FM was the principal investigator in Italy and recruited patients. PdNB was the principal investigator in Denmark and recruited patients. JA and KC contributed to data monitoring. FH contributed to study design. NM contributed to study oversight and data monitoring. SS contributed to data interpretation and recruited patients. MN, GW, BG, NS, and LTr contributed to study design and recruited patients. BA contributed to statistical analysis and data interpretation. MP, GH, MW-H, LTh, PB, AV, MS, UK, CS, RMah, RMar, H-GH, BM, JD, NF, $\mathrm{MH}, \mathrm{AN}, \mathrm{MK}, \mathrm{AT}, \mathrm{MF}, \mathrm{EL}, \mathrm{AdR}, \mathrm{RT}$, and MM recruited patients. All authors have reviewed and approved the final version of the report.

\section{Declaration of interests}

VP and HH report grants from the non-profit organisation Deutsche Krebshilfe; $\mathrm{HH}$ and $\mathrm{MN}$ report grants from Roche; and UK and MN report personal fees from Roche, during the conduct of the study. VP reports personal fees from Hexal and non-financial support from AbbVie, Amgen, and Roche. GH reports personal fees from Bristol-Myers Squibb (BMS), Roche, Amgen, Spectrum, and Merck Sharp \& Dohme (MSD) and grants from BMS and Roche. AV reports personal fees from Roche, Kite/Gilead, Pfizer, BMS, and Amgen. CS reports personal fees from Novartis AG, Gilead, and Takeda and grants and non-financial support from Janssen Cilag, Gilead, and Takeda. LTr reports personal fees from Takeda. UK reports personal fees from Janssen Cilag, BMS, Gilead, Takeda, Amgen, Hexal, Celgene, AstraZeneca, and MSD; advisory board, travel support, and speaker honoraria from Janssen Cilag, BMS, Takeda, Celgene, and MSD; advisory board and travel support from Gilead; and speaker honoraria from Amgen. RM reports personal fees from Roche, Servier, and Merck. MK reports personal fees from AstraZeneca. FM reports personal fees from Roche, Takeda, Celgene, Janssen, Gilead, and Mundipharma and non-financial support from Takeda and Celgene. PdNB reports personal fees from Roche. FH reports personal fees from BMS, Roche, Boehringer Ingelheim, Sanofi, Celgene, Novartis, Ipsen, Janssen, and Gilead. SS reports personal fees, grants, and non-financial support from AbbVie, Amgen, AstraZeneca, Celgene, Gilead, GlaxoSmithKline (GSK), Roche, Janssen, Novartis, Pharmacyclics, and Sunesis. MN reports personal fees and grants from Roche. BG reports personal fees from Roche, Janssen Cilag, Celgene, and Sandoz/Hexal, grants from Roche and Celgene, non-financial support from Roche, and travel expenses from Roche, Janssen Cilag, and Sandoz/Hexal. RT reports grants from Roche; advisory board for AbbVie and Atara; and travel support for Celgene, Janssen, Gilead, and AbbVie. All other authors declare no competing interests.

\section{Data sharing}

Individual data will be made available as well as data dictionaries. Data that underlie the main results reported in this Article, will be shared after de-identification. The study protocol is available in the appendix (pp 49-160). Pseudonymised data will be available upon request up to 5 years after publication of this Article to researchers who provide a data sharing agreement that describes a methodically sound proposal and the data necessary for the purpose of the approved proposal. Proposals should be directed to dshnhl@uks.eu. Data will be shared once all relevant parties approve and sign the data sharing agreement. Selected data will be also available on the Leipzig Health Atlas website.

\section{Acknowledgments}

The trial was supported by a grant from the German non-profit foundation, Deutsche Krebshilfe (German Cancer Aid), reference number 106369. We thank the patients, families, caregivers, and principal investigators of all countries who participated in the clinical trial including PdNB (Denmark), HH (Norway); FM (Italy); OS (Israel); and MP (Germany), who designed the study, wrote the protocol, was the principal investigator in Germany, and was chairman of the study. We thank the numerous research and trial groups, including the
For the Leipzig Health Atlas website see www.health-atlas.de 
German High-Grade Non-Hodgkin's Lymphoma Study Group, the Fondazione Italiana Linfomi, and the Nordic lymphoma group for their participation in the trial. We thank the international board of expert pathologists who provided histopathological review: Andreas Rosenwald (chairman; Wuerzburg, Germany); Jan Delabie (Oslo, Norway); Alfred C Feller (Luebeck, Germany); Martin-Leo Hansmann (Frankfurt, Germany); Wolfram Klapper (Kiel, Germany); Peter Moeller (Ulm, Germany); Hans Konrad Mueller-Hermelink (Wuerzburg, Germany); Harald Stein (Berlin, Germany); Christer Sundstroem (Uppsala, Sweden); Philippe Trougouboff (Afula, Israel); and Hans-Heinrich Wacker (Kiel, Germany). We thank Christian Berdel, Jochen Fleckenstein, and Christian Ruebe for imaging review as independent expert radiologists at Homburg/Saar, Germany. We thank the data and safety monitoring committee that served as an independen expert advisory group to assess safety and efficacy data during the trial, including Günter Brittinger (Essen, Germany); Volker Diehl (Koeln, Germany); and Klaus Havemann (Marburg, Germany; died May, 2016) We thank the study trial office Homburg/Saar, Germany, including the secretaries Waltraud Beck (died August, 2013) and Daniela Ehlert; the data management team Stephanie Angel, Elina Dick, Kerstin Höhn, Kirstin Monz, Tanja Rixecker, and Christian Schorpp; and the clinicians Josif Amam, Konstantinos Christofyllakis, Gerhard Held, Niels Murawski, Milena Pfeiffer, Viola Poeschel, Christoph Renner, Rudolf Schmits (died October, 2016), Jörg Schubert, Pia Sweet, Anne Wolf, and Carsten Zwick. We thank the data centre in Leipzig, Germany, including the database team Sigrid Haupt, Jürgen Hentschel, Martina Kunert, Beate Mann, Katja Rillich, Ulrike Schoenwiese, and Barbara Wicklein; and the biometry team Bettina Altmann, Markus Loeffler, and Marita Ziepert.

References

1 Coiffier B, Lepage E, Brière J, et al. CHOP chemotherapy plus rituximab compared with $\mathrm{CHOP}$ alone in elderly patients with diffuse large-B-cell lymphoma. N Engl J Med 2002; 346: 235-42.

2 Pfreundschuh M, Trümper L, Österborg A, et al. CHOP-like chemotherapy plus rituximab versus CHOP-like chemotherapy alone in young patients with good-prognosis diffuse large-B-cel lymphoma: a randomised controlled trial by the MabThera International Trial (MInT) Group. Lancet Oncol 2006; 7: 379-91.

3 Pfreundschuh M, Schubert J, Ziepert M, et al. Six versus eight cycles of bi-weekly CHOP-14 with or without rituximab in elderly patients with aggressive CD20+ B-cell lymphomas: a randomised controlled trial (RICOVER-60). Lancet Oncol 2008; 9: 105-16.

4 International Non-Hodgkin's Lymphoma Prognostic Factors Project. A predictive model for aggressive non-Hodgkin's lymphoma. N Engl J Med 1993; 329: 987-94.

5 Ziepert M, Hasenclever D, Kuhnt E, et al. Standard International prognostic index remains a valid predictor of outcome for patients with aggressive CD20+ B-cell lymphoma in the rituximab era. J Clin Oncol 2010; 28: 2373-80.

6 Pfreundschuh M, Ho AD, Cavallin-Stahl E, et al. Prognostic significance of maximum tumour (bulk) diameter in young patients with good-prognosis diffuse large-B-cell lymphoma treated with CHOP-like chemotherapy with or without rituximab: an exploratory analysis of the MabThera International Trial Group (MInT) study. Lancet Oncol 2008; 9: 435-44.

7 Piantadosi S. Clinical Trials. Chichester, UK: John Wiley \& Sons, 1983: 213-14

8 Boehme V, Schmitz N, Zeynalova S, Loeffler M, Pfreundschuh M. CNS events in elderly patients with aggressive lymphoma treated with modern chemotherapy (CHOP-14) with or without rituximab: an analysis of patients treated in the RICOVER-60 trial of the German High-Grade Non-Hodgkin Lymphoma Study Group (DSHNHL). Blood 2009; 113: 3896-902.

9 Cheson BD, Horning SJ, Coiffier B, et al. Report of an international workshop to standardize response criteria for non-Hodgkin's lymphomas. J Clin Oncol 1999; 17: 1244.

10 Makuch R, Simon R. Sample size requirements for evaluating conservative therapy. Cancer Treat Rep 1978; 62: 1037-40.

11 Rothmann MD, Wiens BL, Chan ISF. Design and analysis of non-inferiority trials, 1st edn. New York: Chapman and Hall/CRC 2012: 381 .
12 Cheson BD, Pfistner B, Juweid ME, et al. Revised response criteria for malignant lymphoma. J Clin Oncol 2007; 25: 579-86.

13 Wunderlich A, Kloess M, Reiser M, et al. Practicability and acute haematological toxicity of 2- and 3-weekly CHOP and CHOEP chemotherapy for aggressive non-Hodgkin's lymphoma: results from the NHL-B trial of the German High-Grade Non-Hodgkin's Lymphoma Study Group (DSHNHL). Ann Oncol 2003; 14: 881-93.

14 Jaeger U, Trneny M, Melzer H, et al. Rituximab maintenance for patients with aggressive B-cell lymphoma in first remission results of the randomized NHL13 trial. Haematologica 2015; 100: 955-63.

15 Pfreundschuh M, Poeschel V, Zeynalova S, et al. Optimization of rituximab for the treatment of diffuse large B-cell lymphoma (II): extended rituximab exposure time in the SMARTE-R-CHOP-14 trial of the german high-grade non-Hodgkin lymphoma study group. J Clin Oncol 2014; 32: 4127-33.

16 Persky DO, Unger JM, Spier CM, et al. Phase II study of rituximab plus three cycles of CHOP and involved-field radiotherapy for patients with limited-stage aggressive B-cell lymphoma: Southwest Oncology Group study 0014. J Clin Oncol 2008; 26: 2258-63.

17 Lamy T, Damaj G, Soubeyran P, et al. R-CHOP 14 with or without radiotherapy in nonbulky limited-stage diffuse large B-cell lymphoma. Blood 2018; 131: 174-81.

18 Cheson BD, Fisher RI, Barrington SF, et al. Recommendations for initial evaluation, staging, and response assessment of Hodgkin and non-Hodgkin lymphoma: the Lugano classification. J Clin Oncol 2014; 32: 3059-68.

19 Casasnovas R-O, Meignan M, Berriolo-Riedinger A, et al. SUVmax reduction improves early prognosis value of interim positron emission tomography scans in diffuse large B-cell lymphoma. Blood 2011; 118: 37-43.

20 Burggraaff CN, de Jong A, Hoekstra OS, et al. Predictive value of interim positron emission tomography in diffuse large B-cell lymphoma: a systematic review and meta-analysis. Eur J Nucl Med Mol Imaging 2019; 46: 65-79.

21 Pfreundschuh M, Murawski N, Christofyllakis K, et al. Excellent outcome of elderly patients with favourable-prognosis DLBCL treated with 4 cycles CHOP/Chlip-14 plus 8 applications of rituximab and a PET-based intensification strategy that includes involved-site radiotherapy (IS-RT): results of the first 120 patients of the OPTIMAL $>60$ trial of the Dshnhl. Blood 2017; 130 (suppl 1): 1549 (abstr).

22 Dührsen U, Müller S, Hertenstein B, et al. Positron emission tomography-guided therapy of aggressive non-Hodgkin lymphomas (PETAL): a multicenter, randomized phase III trial. J Clin Oncol 2018; 36: 2024-34

23 Rosenwald A, Wright G, Leroy K, et al. Molecular diagnosis of primary mediastinal B cell lymphoma identifies a clinically favorable subgroup of diffuse large B cell lymphoma related to Hodgkin lymphoma. J Exp Med 2003; 198: 851-62.

24 Vitolo U, Chiappella A, Ferreri AJM, et al. First-line treatment for primary testicular diffuse large B-cell lymphoma with rituximabCHOP, CNS prophylaxis, and contralateral testis irradiation: final results of an international phase II trial. J Clin Oncol 2011; 29: 2766-72.

25 Luminari S, Ferrari A, Manni M, et al. long-term results of the FOLL05 trial comparing R-CVP versus R-CHOP versus R-FM for the initial treatment of patients with advanced-stage symptomatic follicular lymphoma. I Clin Oncol 2018; 36: 689-96.

26 Maurer MJ, Ghesquières H, Jais J-P, et al. Event-free survival at 24 months is a robust end point for disease-related outcome in diffuse large B-cell lymphoma treated with immunochemotherapy. J Clin Oncol 2014; 32: 1066-73.

27 Schmitz N, Zeynalova S, Nickelsen M, et al. CNS internationa prognostic index: a risk model for CNS relapse in patients with diffuse large B-cell lymphoma treated with R-CHOP. J Clin Oncol 2016; 34: 3150-56.

28 Bartlett NL, Wilson WH, Jung S-H, et al. dose-adjusted EPOCH-R compared with R-CHOP as frontline therapy for diffuse large B-cell lymphoma: clinical outcomes of the phase III Intergroup Trial Alliance/CALGB 50303. J Clin Oncol 2019; 37: 1790-99.

29 Vitolo U, Trněný $M$, Belada $D$, et al. obinutuzumab or rituximab plus cyclophosphamide, doxorubicin, vincristine, and prednisone in previously untreated diffuse large B-cell lymphoma. J Clin Oncol 2017; 35: 3529-37. 
Articles

30 Younes A, Sehn LH, Johnson P, et al. Randomized phase III trial of ibrutinib and rituximab plus cyclophosphamide, doxorubicin, vincristine, and prednisone in non-germinal center B-cell diffuse large B-cell lymphoma. J Clin Oncol 2019; 37: 1285-95.

31 Davies A, Cummin TE, Barrans S, et al. Gene-expression profiling of bortezomib added to standard chemoimmunotherapy for diffuse large B-cell lymphoma (REMoDL-B): an open-label, randomised, phase 3 trial. Lancet Oncol 2019; 20: 649-62.
32 Copie-Bergman $\mathrm{C}$, Cuillière-Dartigues $\mathrm{P}$, Baia $\mathrm{M}$, et al

MYC-IG rearrangements are negative predictors of survival in

DLBCL patients treated with immunochemotherapy: a GELA/LYSA

study. Blood 2015; 126: 2466-74. 